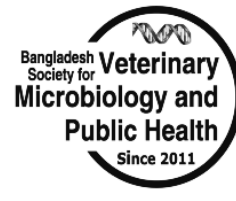

\title{
A Cross Sectional Study on Prevalence of Bovine Tuberculosis of Buffaloes in Bangladesh
}

\author{
Md. Lokman Hossain, Mohammad Ferdousur Rahman Khan*, K.H.M. Nazmul Hussain Nazir and Md. Bahanur \\ Rahman
}

Department of Microbiology and Hygiene, Faculty of Veterinary Science, Bangladesh Agricultural University, Mymensingh-2202, Bangladesh.

*Corresponding author's e-mail: frkhanbau80@yahoo.com

[Received: 12 May 2012, Revised: 20 May 2012, Accepted: 20 June 2012]

\begin{abstract}
A B S T R A C T
This study was conducted to determine the prevalence of bovine tuberculosis in buffalo population in Bangladesh by Caudal Fold Tuberculin (CFT) test using bPPD and also by Comparative Cervical Tuberculin (CCT) test using both bPPD and aPPD. The percentage of reactors was $6.12 \%$ in CFT, whereas the percentage of positive buffaloes was $4.08 \%$ by CCT method. The percentage of suspicious buffaloes was $10.20 \%$ by CFT. On the other hand, all the suspicious cases were proved as misleading-false-reactions in CCT. By CCT method, the male buffaloes $(6.25 \%)$ showed more positivity to tuberculosis than the female buffaloes $(3.03 \%)$ of the same area. In contrast, the result found by CFT method was more or less similar (20\%) for both male and female buffaloes. Young buffaloes (6-30 months) were more susceptible to tuberculosis than older buffaloes. It might be suggested that a well coordination in activities should be taken among the public health and Veterinary public health organelles for complete eradication of the disease from the country.
\end{abstract}

Keywords: Bovine Tuberculosis, Caudal Fold Tuberculin Test, Comparative Cervical Tuberculin Test, Buffalo

(C) 2012 Microbes and Health. All rights reserved

\section{Introduction}

Bovine tuberculosis is a chronic, contagious respiratory disease of cattle and buffaloes transmitted horizontally within and between species, by the aerosol and ingestion (O'Reilly and Daborn, 1996). Tuberculosis plays a central role in public health and animal health because of its severity in humans, in addition to the economic losses related to affected herds (Rodriguez et al., 1999). Tuberculosis has been recognized at least from 176 countries as being one of the important bovine diseases causing great economic losses (Hines et al., 1995). Mycobacterium tuberculosis, $M$. bovis and M. avium all the three species are capable of causing disease in humans although $M$. tuberculosis is the most common in humans which continues to be a major cause of morbidity and mortality throughout the world.

Buffaloes are a documented reservoir for M. bovis in South Africa from which the pathogen can be transmitted to cattle and other animals (Michel et al., 2007). Prevalence of bovine TB in buffalo can reach up to $92 \%$ as in South Africa (De-Vos et al., 2001). In Indo-Pak, the incidence of Bovine tuberculosis in cattle and buffalo is nearly $2.25 \%$ of the population in 1969 (Lall, 1969) and in 1989 (Shahid, 1989), the incidence of disease was increased up

To cite this article: Hossain ML, MFR Khan, KHMNH Nazir and MB Rahman, 2012. A cross sectional study on prevalence of bovine tuberculosis of buffaloes in Bangladesh, 1(1): 23-26. to $7.3 \%$ in cattle and buffaloes being slaughtered at Lahore abattoirs. In 1975, it was reported that $10 \%$ of the buffalo population was suffering from Bovine Tuberculosis in Pakistan (AVA, 1975). The prevalence of tuberculosis in buffaloes at the Agriculture Development Cooperation Farm Rohri and Quetta, Pakistan, as reported by Khilji (1974) was 0.53 and $5.31 \%$, respectively. The prevalence of the disease in buffaloes within a $10 \mathrm{~km}$ radius around Faisalabad city, Pakistan, was reported to be $1.76 \%$ (Ifrahim, 2001), while it was reported to be as high as 30$40 \%$ in India (Guha and Sarkar, 1970) and 20.2\% in Egypt (Guindi et al., 1981). M. bovis, the cause of bovine tuberculosis and M. tuberculosis, the cause of classical human tuberculosis, are genetically and antigenically very similar and cause identical clinical disease in humans (Dankner $e t$ al., 1993).

Many methods are available for diagnosis of tuberculosis in infected animals but the single comparative intradermal tuberculin test (SCITT) is most widely used for diagnosis and eradication of Bovine tuberculosis (OIE, 1999). In Bangladesh, so far the single intradermal (SID) skin test with purified protein derivative (PPD) has been used to detect the prevalence of bovine TB (BTB), and an overall $5.9 \%$ cattle in the district of Pabna (Pharo et al., 1981), $3.05 \%$ cattle in the district of Mymensingh (Samad and Rahman, 1986) and $27.5 \%$ breeding bulls (Islam et al., 2007) showed positive reaction to the tuberculin test. Sero-diagnostic tests, ICGA as Antigen Rapid Bovine TB $\mathrm{Ab}$ Test Kit has been used for the first time in Bangladesh to detect the prevalence of BTB and its effect on milk production in lactating cows (Samad and Rahman, 2008). 
As evident from the preceding discussion, the prevalence of the disease varies from region to region and even from one farm to another in same locality. Countries known to be disease-free have spent years working hard through continuous monitoring and test and slaughter policies. Unfortunately, bovine TB has never been taken seriously in Bangladesh. Sufficient information regarding its incidence in buffaloes and its economic impact on livestock industry is not available. There is little empirical data regarding how the prevalence of infection changes over time in large, free-ranging mammals. No serious efforts are underway to estimate the gravity of risk and its threat to the public health. The existing situation calls for a comprehensive program to address TB in livestock species including buffaloes in order to improve the lot of livestock and to safeguard the human population from this menace. In the present study, the experiments were undertaken aiming to determine the prevalence of bovine tuberculosis in buffalo population in Bangladesh by CFT test using bPPD and also by CCT test using both bPPD and aPPD

\section{Materials and Methods}

A total of 49 buffaloes at Bangladesh Livestock Research Institute (BLRI) farm were tested by CFT test using bovine PPD at the caudal fold and the reaction of the buffaloes was observed. All the reactors and suspected buffaloes to CFT were subjected to CCT test using both bovine (bPPD) and avian PPD (aPPD) at the two sites of the neck region for confirmation. The data were recorded in a questionnaire and finally analyzed to determine the prevalence and risk factors associated with the prevalence of bovine and avian tuberculosis in buffaloes.

\section{Selection and grouping of animals}

Forty nine (49) buffaloes kept at BLRI buffalo farm, Savar, Dhaka were selected to determine the prevalence of tuberculosis using CFT and CCT. Of the 49 buffaloes, 6 were milking buffaloes, 10 dry buffaloes, 7 heifer, 5 male calves, 11 bulls and 10 female calves. Date of birth and managemental information were recorded in a questionnaire from the register book of the farm.

\section{Selection of tuberculin and other necessary materials and instruments}

For the CFT testing, bovine PPD and for the CCT testing, both the bovine PPD and avian PPD were used in this study. Both the PPDs were obtained from a licensed laboratory in Italy (Instituto Zooprofilattico sperimentale dell'Umbria e delle Marche, Periegia). All the tuberculin vials were kept in a refrigerator for maintaining the potency at quality level. For the purpose of cleaning and disinfection of the inoculation site, sterilized cotton and $70 \%$ ethyl alcohol were used. For inoculation, $1 \mathrm{ml}$ tuberculin syringe of 100 graduations, fitted with a short hypodermic needle was utilized

\section{CFT test and CCT test}

Each and every animal was inoculated two times (CFT and $\mathrm{CCT}$ ) as required by these procedures. The initial caudal fold inoculation was done with an equivalent dosage of 10,000 tuberculin unit/ml of bovine type and after a period of 10 days the Comparative cervical inoculation was made by $10,000 \mathrm{TU} / \mathrm{ml}$ of bovine and 2,500 TU $/ \mathrm{ml}$ of avian type of separate way on one side (upper and lower) of the neck region. The method described by Welchi (1915) was followed throughout the experiment for inoculating the animals. As per described method, the animal was first restrained and the tail was secured by properly trained assistants and the hair free and pliable area of caudal fold was cleaned properly and aseptically by gently rubbing with sterilized cotton, soaked in $70 \%$ ethyl alcohol. Then the inoculation was made at the point where the caudal fold merges with the tail. In the initial caudal fold testing $0.1 \mathrm{ml}$ (10 graduations of the syringe) of bovine PPD, containing 10,000 TU/ml was pushed slowly under the epidermis to a horizontal way in one side of the caudal fold by securing it in between the thumb and the $1^{\text {st }}$ and $2^{\text {nd }}$ finger. After a period of 10 days, following the same procedure of inoculation, $0.2 \mathrm{ml}$ of avian PPD was given in the upper side of the neck. Another $0.1 \mathrm{ml}$ of bovine PPD was inoculated in the lower side of the neck.

\section{Reading of the results of inoculations}

After the inoculation was made, the reading was taken for one time after 72 hours. The positive tuberculin reaction was evident from an inflammation of sensitive nature at the point of inoculation. The area of the characteristic swelling ranged from the size of a small pea to that of an orange. The reaction sites indicated the organism responsible for the sensitization. The swelling was either soft and edematous or somewhat hard in nature. The swelling was estimated by palpation at the site of inoculation, while the animal showed the signs of pain.

\section{Statistical analysis of the result}

The collected data was compiled, tabulated and analyzed in accordance with the objectives of the study. The approximate percentage was calculated for each parameter. Finally data were analyzed using the statistical software 'SPSS' by chi-square tests for the relationship of different factors on the occurrence of tuberculosis in buffaloes where $p$ value of $\leq 0.5$ was considered statistically significant.

\section{Results}

In CFT test, reading was recorded once after 72 hours of the inoculation of bPPD. The positive CFT was evident from an inflammation of sensitive nature at the point of inoculation and the area of the characteristic swelling ranged from- the size of a small pea to that of an orange (Fig. 1). The swelling was either soft and edematous or somewhat hard in nature. In CCT test, two injections were made intradermally; bPPD at upper site and aPPD at lower site at neck region. Initial skin thickness was measured before inoculation PPD. Reading was taken after $72( \pm 6)$ hours with slide calipers. A swelling of $\geq 4 \mathrm{~mm}$ from the initial reading indicated positive. On the other hand, a swelling of 3.0-3.99 $\mathrm{mm}$ from the initial reading indicated doubtful and $<3.0 \mathrm{~mm}$ indicated as negative (Fig. 2).

\section{Results of CFT test using bPPD}

In this study, 49 buffaloes of different sex, age and types were examined to determine the incidence of tuberculosis by CFT test. Out of 49 buffaloes, $3(6.12 \%)$ reacted to CFT test with bPPD showing marked painful swelling of the inoculation site at the caudal fold, and $5(10.20 \%)$ buffaloes showed suspicious reaction with the test which showed only slight swelling at the site of inoculation and the remaining 41 buffaloes (83.67\%) did not react showing no swelling at the site of inoculation. 


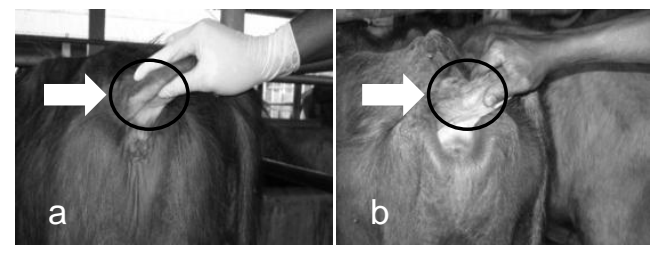

Fig. 1: Reactor buffalo showing marked swelling (a. positive reaction) and no change (b. negative reaction) of the inoculation site at caudal fold in CFT test.

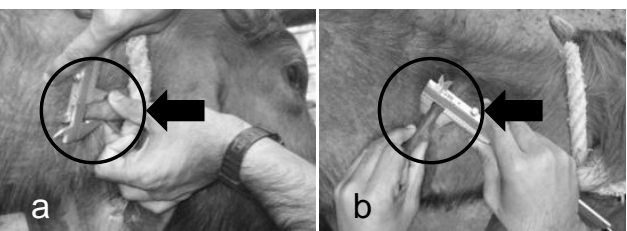

Fig. 2: Reactor buffalo showing increased thickness (a. positive reaction) and no change (b. negative reaction) at skin of neck in CCT test.

\section{Results of CFT test on the basis of sex}

(i) Reactor buffaloes based on sex

Of the 49 buffaloes which were subjected to CFT test, the number of male reactor was only one $(6.25 \%)$ while the female reactors were $2(6.06 \%)$.

(ii) Suspicious reactor buffaloes based on sex

Of the 49 buffaloes which were subjected for CFT test, the number of suspicious male reactors was $2(12.6 \%)$ while the numbers of suspicious female reactors were $3(9.09 \%)$ in the same test.

\section{Results of CFT test on the basis of age}

Among the 49 buffaloes of different age those were subjected to initial CFT test, highest percentage (14.29\%) of reactor buffaloes and also suspicious buffaloes were observed in the age group between 2 years and 6 months to 4 years and 6 months followed by the 6 months to 2 year and 6 months age group of buffalo $(7.14 \%$ reactor and $10.71 \%$ suspicious cases). No reactor to CFT test was found in buffaloes of more than 4 years and 6 months of age although $12.5 \%$ suspicious cases were recorded.

\section{Result of CFT test on the basis of types of animals}

Of the 49 buffaloes, highest percentage (20\%) of reactors of CFT test were found in male calves followed by $14.29 \%$ in heifer and $10 \%$ in female calves, whereas the highest percentages of suspicious cases $(20 \%)$ were found in both male and female calves followed by buffalo bulls $(9.09 \%)$ in CFT test using bPPD.

\section{Results of CCT test using bPPD and aPPD}

All the reactors and suspected buffaloes at BLRI farm were subjected to CCT test using both aPPD and bPPD at two sites of neck (upper and lower sites, respectively). Out of 8 buffaloes, only 2 showed an increase in the thickness of skin for about $4 \mathrm{~mm}$ at the site of inoculation (positive reaction) of bovine $\mathrm{PPD}$ on the neck region and the remaining buffaloes showed no reaction to CCT test. Also, there was no reaction found for avian PPD.

\section{(i) Results of CCT test on the basis of sex}

Among the 49 buffaloes, only 1 male buffalo with a percentage of 6.25 were confirmed by CCT test as TB infected, on the other hand, it was found only in 1 female buffalo with a percentage of 3.03 . (ii) Results of CCT test on the basis of age

Among the 49 buffaloes of different age those were subjected CCT test, 2 buffaloes with a percentage of 7.14 were found as positive in the age group between 6 to 30 months while no other buffalo was found positive by CCT test in the current study.

(iii) Results of CCT test on the basis of types of buffaloes Of the 49 buffaloes under study, highest percentage (20\%) of positive buffaloes in CCT were found in male calves followed by $10 \%$ in female calves. On the other hand, no other buffalo was revealed as positive by CCT test among the heifers, buffalo bulls, milking and dry buffaloes.

\section{Discussion}

The present study was conducted to determine the prevalence of bovine and avian tuberculosis among the buffaloes of BLRI buffalo farm, Savar by CFT test using bPPD and also by CCT test using both bPPD and aPPD. The intradermal tuberculin test was performed according to the method described by Welchi (1915). In the CFT testing, some of the reactions $(6.12 \%)$ were evident from a clear inflammatory response like swelling of painful nature, while in other cases (10.20\% slight swelling) lead to suspicious. The reactions which were not clear after 72 hours of inoculation were recorded as negative (83.67\%). During the CCT testing after 10 days of the CFT testing, all the suspicious cases were proved as misleading-false-reactions. Similar finding was reported by Lopes et al. (2006) who found variations in the numbers of positive animals between the CCT and CFT tests.

The percentages of reaction to tuberculin tests in buffaloes were found moderately low in this study. The percentage of positive reaction was $4.08 \%$ in CCT test which is in agreement with the findings of Javed et al. (2006) who reported that the prevalence of tuberculosis in buffaloes on the basis of comparative intradermal tuberculin test. They found that it varies from as high as $8.48 \%(14 / 165)$ to as low as $2.45 \%(4 / 163)$. The results in the current study is also in agreement with the findings of Rodwell et al. (2001) who estimated the prevalence of BTB as 0 and $4.4 \%$ in the north and central area of Kruger National Park (KNP), respectively. On the other hand, positive reaction $(4.08 \%)$ obtained in this study was much lower than those obtained by Cataluna and Gordoncillo (2006) who found $38 \%$ of the water buffaloes to react positively with tuberculin test. So, it is difficult to draw any conference as a whole on the incidence of tuberculosis in the buffalo under this study, as the rate of incidence of tuberculosis is influenced by many factors such as geographical situation of a country, temperature, precipitations, hygienic status of humans and animals, and enforced regularity laws in Public Health and Veterinary Public Health sectors. However, the incidence seems to be reasonably low in the present experimental area. No program has been undertaken as yet to eradicate tuberculosis from the animals particularly Buffaloes in Bangladesh. Though a low percentage of positive reactors were found in this study, however, it would not be wise to make delay to take control measures in Bangladesh.

In the tuberculin testing, the male buffaloes showed higher percentage $(6.25 \%)$ of reaction than the female buffaloes (3.03\%) of the same area by CCT while the percentage of 
reactor buffaloes was similar $(20 \%)$ in both male and female buffaloes with CFT test. Similar finding was reported by TRC (2003) who noted a substantially higher incidence of BTB in males $(8.6 \%)$ than females $(3.1 \%)$. The percentage of reactor animals in relation with sex in buffalo is insignificant $(p>0.05)$ as detected by CCT.

In terms of age, buffaloes between 2 years and 6 months to 4 years and 6 months of age found more $(14.29 \%)$ susceptible to tuberculosis than the buffaloes of other ages in CFT. This figure was highest $(7.14 \%)$ in buffaloes between 6 to 30 months of age by CCT. The correlation in different age groups for the prevalence of tuberculosis is insignificant in buffalo $(p>0.05)$. When the type of buffaloes was considered, the male calves showed highest percentage $(20 \%)$ of positivity to CCT followed by female calves $(10 \%)$. However, the differences were insignificant $(p>0.05)$ in the percentage of positive animals by CCT when different age and sex of buffaloes were considered.

\section{Conclusion}

Forty nine buffaloes of different age, sex and type were examined to determine the prevalence of tuberculosis by CFT and CCT test using bovine PPD and avian PPD obtained from a licensed laboratory in Italy. By CFT test, the percentage of positive reaction (reactors) was 6.25 , whereas the percentage was 4.08 by CCT. The overall percentages of suspicious cases were 10.20 in CFT while no suspicious cases were found in CCT. In general, the young buffaloes (6-30 months) were infected higher than the older buffaloes as detected in this study. However, the overall infection rate detected by the methods employed in this study was lower than that of other studies in relevant published literatures.

\section{Acknowledgement}

Special thanks to Dr. Monica Cagiola (Director, Instituto Zooprofilacttico Sperimentale dell' Umbria e delle Marche, Perugia, Italy), for providing bPPD and aPPD.

\section{References}

AVA, 1975. Australian Veterinary Association, Bovine tuberculosis: Report to the Australian Industries Assistance Commission, pp: 7.

Cataluna RLN and MJN Gordoncillo, 2006. Cervical fold tuberculin testing in cattle (Bos indicus) and water buffaloes (Bubalus bubalis) in Los Banos, Laguna. Philippine J Vet Med, 43: 103-105.

Dankner WM, NJ Waecker, MA Essey, K Moser, M Thompson and CE Davis, 1993. Mycobacterium bovis infections in San Diego: a clinicoepidemiologic study of 73 patients and a historical review of a forgotten pathogen. Med (Baltimore), 72: 11-37.

De-Vos V, RG Bengis, NP Kriek, A Michel, DF Keet, JP Raath and HF Huchzermeyer, 2001. The epidemiology of tuberculosis in free-ranging african buffalo (Syncerus caffer) in the Kruger National Park, South Africa. Onders J Vet Res, 68: 119-130.

Guha AN and PB Sarkar, 1970. Study of tuberculosis amongst cattle in Calcutta. Ind Vet J, 47: 196.

Guindi S, MO Lofty and MW Awad, 1981. Some observations regarding the infectivity and sensitivity for tub- erculosis in buffaloes in Arab Republic of Egypt. J Egy Vet Med Assoc, 35: 125-138.

Hines ME, JM Kreeger and PK Smith, 1995. Microbacterial infections of animals. Pathology and Pathogenesis. Lab Anim Sci, 45: 334-351.

Ifrahim M, 2001. Epidemiological studies on tuberculosis in cattle and buffalo population in villages around Faisalabad. M. Sc. (Hon.) Thesis, Department of Veterinary Microbiology, University of Agriculture, Faisalabad, Pakistan.

Islam MM, MAR Siddique, MA Haque, MA Baki, S Majumder, JJ Parrish and M Shamsuddin, 2007. Screening some major communicable diseases of AI bulls in Bangladesh. Lives Res Rur Dev, 19: 79.

Javed MTM, M Usman, M Irfan and M Cagiola, 2006. A study on tuberculosis in buffaloes: some epidemiological aspects, along with haematological and serum protein changes. Vet Arhiv, 76: 193-206.

Khilji IA, 1974. Incidence of tuberculosis among Kundi buffaloes. Pak J Anim Sci, 13: 27-31.

Lall JM, 1969. Tuberculosis among animals in India. Vet Bull, 39: 316-324.

Lopes LB, AP Cunha, RA Mota and RC Leite, 2006. Comparison of two tuberculin tests in buffaloes. Cienc Anim Brasil, 7: 187-191.

Michel AL, LM Klerk, NG Pittius, RM Warren and PD Helden, 2007. Bovine tuberculosis in African buffaloes: observations regarding Mycobacterium bovis shedding into water and exposure to environmental mycobacteria. BMC Vet Res, 3: 23.

O'Reilly LM and CJ Daborn, 1995. The epidemiology of Mycobacterium bovis infections in animals and man: a review. Tuber Lun Dis, 76: 1-46.

OIE, 1999. Office International des Epizooties: Manual of Diagnostic Tests and Vaccines for Terrestrial Animals: Bovine tuberculosis, pp: 1-9.

Pharo HJ, A Motalib, SF Routledge and S Alam, 1981. The prevalence of bovine tuberculosis in Bangladesh; Cattle development project. Bangl Vet J, 15: 53-56.

Rodriguez JG, JC Fissanoti, P Del Portillo, ME Patarroyo, MI Romano and A Cataldi, 1999. Amplification of a 500-basepair fragment from cultured isolates of Mycobacterium bovis. Eur J Clin Microbiol Infect Dis, 37: 2330-2332.

Rodwell TC, NP Kriek, RG Bengis, IJ Whyte, PC Viljoen, V de-Vos and WM Boyce, 2001. Prevalence of bovine tuberculosis in African buffalo at Kruger National Park. J Wildl Dis, 37: 258-264.

Samad MA and MS Rahman, 2008. Prevalence of bovine tuberculosis and its effects on milk production in Red Chittagong cattle. Bangl J Vet Med, 6: 175-178.

Samad MA and MS Rahman, 1986. Incidence of bovine tuberculosis and its effect on certain blood indices in dairy cattle of Bangladesh. Ind J Dairy Sci, 39: 3-6.

Shahid A, 1989. Prevalence of buffalo tuberculosis by using short thermal test and identification of organism from lymph nodes. MSc (Hons) Thesis. CVS. University of Agriculture, Faisalabad, Pakistan.

TRC (Tuberculosis Research Centre, India), 2003. Association of initial tuberculin sensitivity, age and sex with the incidence of tuberculosis in south India: a 15year follow-up. Int J Tubercul Lung Dis, 7: 1083-1091.

Welchi H, 1915. The Intradermal tuberculin test. J Am Vet Med, 10: 432. 